\title{
Dynamical conductance of model DNA sequences
}

\author{
Wei Ren and Jian Wang ${ }^{\text {a) }}$ \\ Center of Theoretical and Computational Physics, The University of Hong Kong, Pokfulam Road, \\ Hong Kong, China and Department of Physics, The University of Hong Kong, Pokfulam Road, \\ Hong Kong, China \\ Zhongshui Ma \\ Department of Physics, Beijing University, Beijing, People's Republic of China 100871 \\ Hong Guo \\ Center for the Physics of Materials, McGill University, Montreal, Quebec H3A 2T8, Canada and \\ Department of Physics, McGill University, Montreal, Quebec H3A 2T8, Canada
}

(Received 10 July 2006; accepted 8 September 2006; published online 24 October 2006)

\begin{abstract}
Using a tight binding model, we have investigated charge transport in model DNA sequences under external ac bias. The numerical results of emittance for several model DNA sequences are found to be well described by an analytical formula, especially when the dynamic response is inductivelike. This formula can be understood from general considerations of scattering matrix theory. The temperature dependence of emittance is also studied numerically within the tight binding model, and dynamic response of the model DNA sequences can change from inductivelike to capacitivelike as temperature is varied. (c) 2006 American Institute of Physics. [DOI: 10.1063/1.2359447]
\end{abstract}

\section{INTRODUCTION}

In 1962, Eley and Splivey suggested that charge transfer through DNA may occur via the overlap of $\pi$ stacks of the base pairs. ${ }^{1}$ Experimental indications of long range charge transfer in DNA molecule were since observed by photoexcitation measurements. ${ }^{2}$ The DNA molecule has a property that its base pairs obey a strict rule: adenine always pairs with thymine while cytosine always pairs with guanine. Such a strict pairing property makes DNA an interesting candidate for fabricating nanoscale structures. ${ }^{3}$ The scientific interest in DNA and, more recently, the perspective of using DNA molecule in nanotechnology ${ }^{3}$ have attracted extensive studies on electric conduction through DNA. ${ }^{4-16}$

Experimental and theoretical reports on charge transport through DNA have provided evidences for a wide range of possibilities, including proximity induced superconductivity, ${ }^{7}$ reasonably good conductor with a resistance of $2.5 \mathrm{M} \Omega,{ }^{5}$ semiconductor behavior with an energy gap of a few $\mathrm{eV}^{6,8}$ and insulating behavior with bias voltage up to $10 \mathrm{~V} \cdot{ }^{4,9,17,18}$ The conducting behavior of DNA appears to depend on many physical and chemical details of the measurements. Since some of these details, such as the presence or absence of an impurity, are difficult to control, theoretically it has been quite useful to focus on some generic features of DNA conduction. ${ }^{10-12,15,16,19,20}$

In this work, we report an investigation of high frequency charge transport in model DNA sequences. We view a DNA chain as a possible and interesting molecular wire. As such, a good understanding of its dynamic response to external high frequency ac bias is desirable. This understanding is certainly necessary if DNA is ever going to be used as components of electronic circuitry. From a theoretical point of

${ }^{a)}$ Electronic mail: jianwang@hkusub.hku.hk view, dynamic response of a molecule is also interesting as it reveals whether the molecule behaves as a capacitive or inductive conductor.

It turns out that understanding high frequency dynamic conductance of molecular scale conductor is an important and general problem of quantum transport theory. When a system is biased by a high frequency external ac potential, dynamic induction may become important for nanoscale conductors whose electronic density of states (DOS) at the Fermi level is small. Dynamic induction gives rise to charge accumulation in the conductor as well as the displacement current; these effects must be considered in a transport theory, otherwise the predicted total current will not be conserved. This issue has been first considered by Büttiker and co-workers and Ma et al. ${ }^{21}$ A related issue is the gauge invariance: transport properties should only depend on the voltage difference of the measuring contacts. ${ }^{21}$ The gauge invariance is satisfied automatically for linear dc response but may become problematic at ac and nonlinear transport. In order to satisfy current conservation and gauge invariance, Coulomb interaction must be considered in the theory which is, actually, the origin of displacement current ${ }^{21}$ in ac situations.

When ac frequency $\omega$ of the external bias is small, the dynamical conductance of a system can be expanded in terms of $\omega$ and the linear coefficient is the emittance $E_{\alpha \beta}$ : $G_{\alpha \beta}(\omega)=G_{\alpha \beta}(0)-i \omega q^{2} E_{\alpha \beta}+O\left(\omega^{2}\right)$, where $\alpha, \beta$ are indices of the device leads and $q$ the charge of the carrier. $E_{\alpha \beta}$ is an important quantity and the sign of the off-diagonal matrix $E_{12}$ tells whether the dynamic response of a two probe system is capacitivelike (negative sign) or inductivelike (positive sign). It is also related to the partial density of states $^{22}$ 
characterizing the DOS for electron scattering. $E_{\alpha \beta}$ has been extensively studied for mesoscopic systems ${ }^{23}$ but its property for a DNA sequence has been unknown.

The rest of the paper is organized as follows. In Sec. II we present the model, theoretical and numerical analysis, as well as results. Section III is a short summary.

\section{MODEL, THEORY, AND RESULTS}

We consider the following tight binding Hamiltonian to describe the model DNA sequence: ${ }^{11-13}$

$$
H=\sum_{l}\left[-t_{\mathrm{DNA}} \cos \left(\theta_{l, l+1}\right)\left(c_{l}^{\dagger} c_{l+1}+\text { H . c . }\right)+\epsilon_{l} c_{l}^{\dagger} c_{l}\right] .
$$

This one dimensional tight binding model has been successfully applied to study a single stranded DNA with result that can be compared with experiment (see Ref. 11). We note that a double stranded DNA would be a realistic model to consider. Here $c_{l}^{\dagger}$ is the creation operator for electron at site $l, \epsilon_{l}$ is the on-site energy which depends on the details of the sequence, $t_{\mathrm{DNA}}$ is the hopping matrix element at zero temperature which describes the $\pi$ - $\pi$ stacking interaction between base pairs, and $\theta_{l, l+1}$ is the randomly fluctuating twist angle between neighboring base pairs. Here we assume that $\theta$ follows a Gaussian distribution such that $\langle\theta\rangle=0$ and $\left\langle\theta^{2}\right\rangle$ $=k T / I \Omega^{2}$ from the equipartition theorem with $I \Omega^{2} / k$ $=250 \mathrm{~K} \cdot{ }^{13,16}$ The on-site energies are chosen as $\epsilon_{\mathrm{A}}$ $=8.24 \mathrm{eV}, \epsilon_{\mathrm{T}}=9.14 \mathrm{eV}, \epsilon_{\mathrm{C}}=8.87 \mathrm{eV}$, and $\epsilon_{\mathrm{G}}=7.75 \mathrm{eV}$ following Ref. 24. For simplicity of analysis, we assume that the model DNA sequence is connected to the outside world through type-G nucleobase so that current can be injected into the DNA sequences. The self-energy due to the electrode is calculated using type-G nucleobase. In the calculation, the hopping matrix element $t_{\mathrm{DNA}}$ is set to $1 \mathrm{eV}$ similar to Ref. 13. The hopping matrix between electrodes and scattering region $t_{d}$ is also set to be $1 \mathrm{eV}$. Since the quantum interference will be destroyed at high temperature, we will restrict numerical calculation to the low temperature regime. We note that the transport measurement of DNA can be carried out for the low temperatures. In fact, the temperature dependence of $I-V$ curve has also been measured from 4 to $300 \mathrm{~K}^{25}$ In this work, we have not studied the effect of environment on ac transport. The influence of environment on dc transport has been studied theoretically before. ${ }^{26}$ Adessi et al. modeled the influence of environment on transport by including the effect of disorder in the tight binding model. They found that if the on-site disorder strength is less than $50 \mathrm{meV}$, the conductance does not decrease much. When the on-site disorder strength is larger than $150 \mathrm{meV}$, however, the conductance can drop by a few orders of magnitude between typical lengths of 10 and $100 \mathrm{~nm}$. For the ac transport, as will be described later, we found that when the dynamic response is inductivelike, there exists an approximated formula to describe the emittance of the system and the inductivelike response can change to the capacitivelike as the temperature is varied. In the presence of environment, as long as the system is in the regime of inductivelike response, our conclusion remains.

\section{A. Emittance}

In this subsection we very briefly review the relevant expressions for calculating the emittance. In the presence of a small ac bias, one can expand ${ }^{21}$ the dynamic conductance matrix to the first order in ac frequency $\omega$ as discussed in Sec. I, i.e.,

$$
G_{\alpha \beta}(\omega)=G_{\alpha \beta}(0)-i \omega q^{2} E_{\alpha \beta}+O\left(\omega^{2}\right) .
$$

Here the first term is the dc conductance $G_{12} \equiv G$, and the second term is the emittance discussed above. $E_{\alpha \beta}$ contains contributions from both the conduction current and the displacement current under ac bias. In the absence of magnetic field, the emittance matrix element $E_{\alpha \beta}$ has been derived before: ${ }^{21}$

$$
E_{\alpha \beta}=\frac{d N_{\alpha \beta}}{d E}-D_{\alpha \beta} .
$$

Here, the global partial DOS $d N_{\alpha \beta} / d E$ describes the density of states for the scattering process that electrons enter from lead $\beta$ and exit to lead $\alpha$. This quantity can be calculated from the spatially resolved local partial DOS,

$$
\frac{d N_{\alpha \beta}}{d E}=\int d x \frac{d n_{\alpha \beta}(x)}{d E},
$$

where the local partial DOS $d n_{\alpha \beta} / d E$ is defined using Green's function ${ }^{28}$ of the Hamiltonian (1):

$$
\frac{d n_{\alpha \beta}(x)}{d E}=\operatorname{Re}\left(\delta_{\alpha \beta}\left[G^{r} \Gamma_{\alpha} G^{r}\right]_{x x}+i\left[G^{r} \Gamma_{\beta} G^{a} \Gamma_{\alpha} G^{r}\right]_{x x}\right) .
$$

The positive quantity $D_{\alpha \beta}$ of Eq. (3) contains the contribution from the displacement current and is given by ${ }^{21,27}$

$$
D_{\alpha \beta}=\int d^{3} x \frac{d n_{\beta}(x) / d E d n_{\alpha}(x) / d E}{d n(x) / d E},
$$

where the injectivity $d n_{\alpha} / d E$ is defined as

$$
\frac{d n_{\beta}(x)}{d E}=\sum_{\alpha} \frac{d n_{\alpha \beta}(x)}{d E}=\left[G^{r} \Gamma_{\beta} G^{a}\right]_{x x},
$$

which describes the local DOS for charged carriers at a space point $x$ which are injected into the system from probe $\alpha$ due to the oscillating chemical potential. The total local DOS is

$$
\frac{d n(x)}{d E}=\sum_{\alpha \beta} \frac{d n_{\alpha \beta}(x)}{d E}=\left[G^{r} \Gamma G^{a}\right]_{x x},
$$

where the total linewidth function is defined as $\Gamma=\Gamma_{L}+\Gamma_{R}$ and the quantity $[\cdots]_{x x}$ denotes the diagonal element of the relevant matrix. Importantly, using the above expressions, it is not difficult to confirm that emittance $E_{\alpha \beta}$ satisfies the current conservation and gauge invariant condition $\Sigma_{\alpha} E_{\alpha \beta}$ $=\Sigma_{\beta} E_{\alpha \beta}=0$. These expressions were derived before ${ }^{21}$ and we refer interested readers to the original papers for more details. In this work, we use them to calculate the emittance for the model DNA sequence.

Using the Fisher-Lee relation, ${ }^{29}$ the global partial DOS can be expressed in terms of scattering matrix $S_{\alpha \beta},{ }^{28}$ 


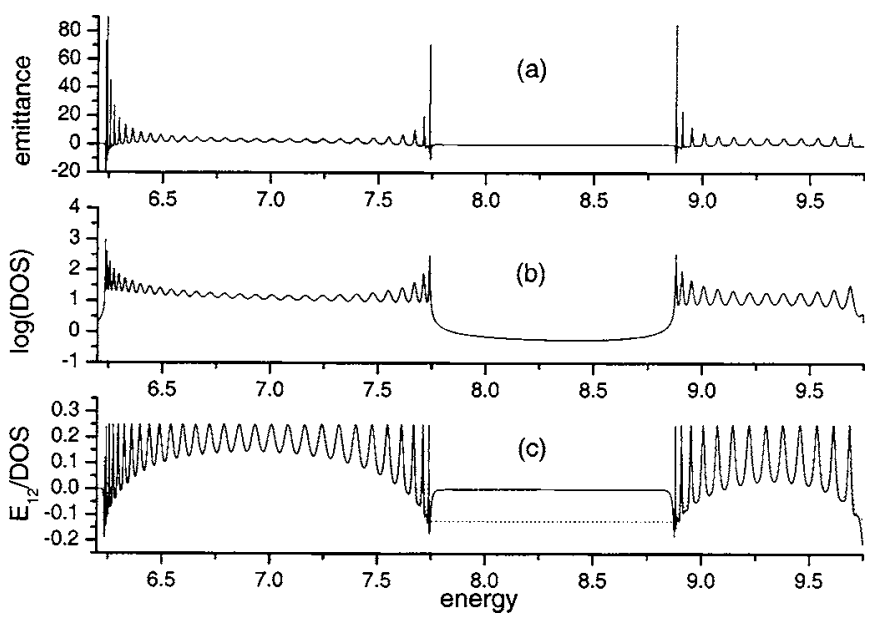

FIG. 1. (a) The emittance (b), logarithm of [global density of states (DOS) (in $\mathrm{eV}$ )], and (c) weighted emittance vs energy for a poly (dGdC) sequence of 60 base pairs (bps). The unit of energy is eV. The unit for emittance is $1 / \hbar \omega$. If we take $\omega=100 \mathrm{MHz}$ then the unit for emittance is $2.5 /(\mu \mathrm{eV})$. The unit for DOS is $1 / \mathrm{eV}$ and the weighted emittance is dimensionless [we have multiplied the weighted emittance in (c) by a factor of $4^{-7}$ ].

$$
\frac{d N_{\alpha \beta}}{d E}=\frac{1}{4 \pi i}\left(S_{\alpha \beta}^{\dagger} \frac{d S_{\alpha \beta}}{d E}-\frac{d S_{\alpha \beta}^{\dagger}}{d E} S_{\alpha \beta}\right) .
$$

As mentioned in Sec. I, two different dynamic behaviors are anticipated depending on the sign of the emittance $E_{12}$. The system shows a capacitivelike behavior if $E_{12}$ is negative. Otherwise, the system is dominated by inductivelike behavior. This can be understood within the picture of scattering matrix theory. ${ }^{21}$ For a capacitor, the dc transmission coefficient is zero, i.e., $S_{12}=0$. Hence $d N_{12} / d E=0$ from Eq. (9). As a result, $E_{12}$ is negative since $D_{\alpha \beta}$ is positive definite [see Eq. (3)]. Then, because $E_{11}+E_{12}=0$ due to current conservation, $E_{11}$ is positive for a capacitor. For a perfect inductor, there is complete transmission so that $S_{11}=0$. Therefore from Eq. (3), $E_{11}$ must be negative. In general, a complete transmission corresponds to an inductivelike dynamic behavior and a complete reflection corresponds to a capacitivelike dynamic behavior. For systems having partial transparency to charge transport, the dynamic response can be either inductive or capacitive, depending on the sign of the emittance.

\section{B. Numerical results}

Using the model DNA sequence Hamiltonian (1), we can easily evaluate Green's function which gives the various DOSs discussed above. The emittance can then be computed numerically.

Figure 1 plots the emittance $E_{12}$, logarithm of global $\mathrm{DOS}=d N / d E$, and $E_{12} / \mathrm{DOS}$ versus energy for a poly-CG sequence. The emittance [Fig. 1(a)] shows many positive peaks which, as we have confirmed, correspond to resonance peaks in the transmission coefficient. This is an inductivelike dynamic behavior. Near the band edges $E \sim 6.2 \mathrm{eV}, 7.7 \mathrm{eV}$, and $8.8 \mathrm{eV}$, these emittance peaks become much higher and narrower due to the large global DOS [Fig. 1(b)]. Interestingly, at these band edges, the emittance can be negative, therefore showing a capacitivelike behavior. Comparing with Figs. 1(a) and 1(b), we observe that the structures of emit-

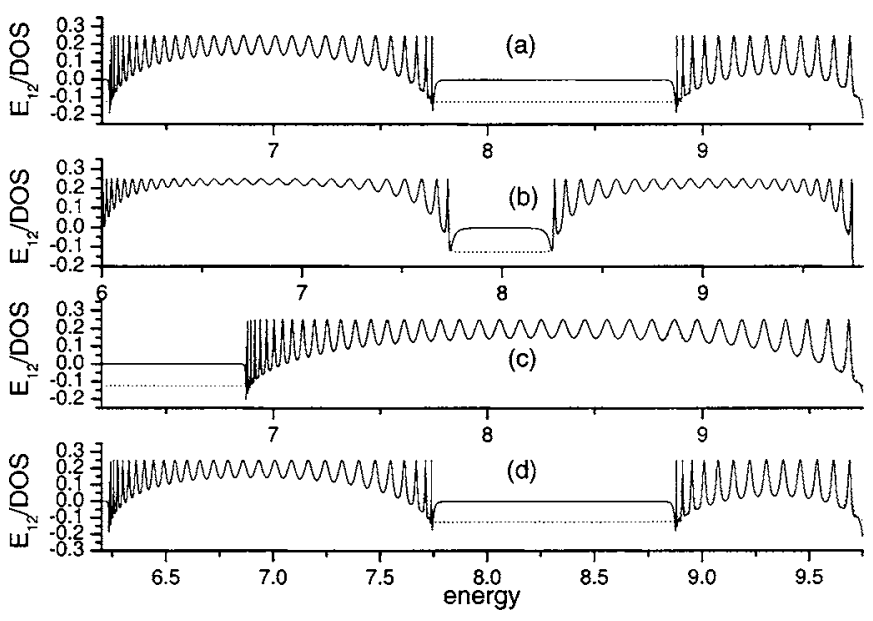

FIG. 2. The weighted emittance (solid) and the quantity $(3 \mathcal{T}-1) / 8$ (dotted) vs energy for poly $(\mathrm{d} \alpha \mathrm{d} \beta)$ sequences of $60 \mathrm{bps}$ : (a) poly(dAdT) sequence, (b) poly (dAdG) sequence, (c) poly (dCdT) sequence, and (d) poly (dGdT) sequence. The units are the same as those of Fig. 1.

tance and the global DOS are well correlated. If we plot $E_{12}$ /DOS versus energy, as in Fig. 1(c), it very much resembles the electron transmission coefficient. In fact, the emittance curves can be nicely fit to the following relation which is a central result of this paper:

$$
E_{12} \approx \frac{3 \mathcal{T}(E)-1}{8} \frac{d N}{d E},
$$

where $\mathcal{T}(E)$ is the transmission coefficient and $d N / d E$ is the DOS. In Fig. 1(c), the solid line is $\bar{E}_{12} \equiv E_{12} /$ DOS and the dotted line is $(3 \mathcal{T}-1) / 8$, showing that Eq. (10) almost holds perfectly. Only near the band edge do we see some small deviations from Eq. (10). We can understand Eq. (10) as follows. It has been shown that ${ }^{22} d n_{12}(r) / d E$ $=(\mathcal{T} / 2) d n(r) / d E$. For a symmetric system, we have approximately $d n_{1} / d E=d n_{2} / d E=(1 / 2) d n / d E$ if we neglect the spatial dependence of $d n_{\alpha} / d E$. We then obtain

$$
E_{12} \approx \frac{2 \mathcal{T}(E)-1}{4} \frac{d N}{d E},
$$

which is rather similar to Eq. (10). The difference between the last two relations is due to the spatial dependence of $d n_{\alpha} / d E$. It is interesting to notice from Fig. 1(c) that all the peak heights in the weighted emittance, $E_{12} / \mathrm{DOS}$, have a value of $1 / 4$, indicating that the resonant points where $\mathcal{T}$ $=1$ are special. At these points, we have $d n_{i i} / d E=0$ for $i$ $=1,2$ from Eq. (9). Hence $d n_{1}(r) / d E=d n_{12}(r) / d E$ $=(1 / 2) d n(r) / d E=d n_{2}(r) / d E$ so that Eqs. (10) and (11) give the same result, $E_{12}=(d N / d E) / 4$. This explains the $1 / 4$ peak heights in Fig. 1(c). However, why Eq. (10) only works for large inductivelike responses is still unclear. When the DNA sequence is well contacted with the electrode and the system is very transmissive, Eq. (10) describes the system very well. Otherwise, the capacitivelike behavior dominates and Eq. (10) fails to describe this behavior.

In Fig. 2 we compare the weighted emittance with Eq. (10) for different model sequences: poly $(\mathrm{d} \alpha \mathrm{d} \beta)$ sequences with $\alpha, \beta=\mathrm{G}, \mathrm{C}, \mathrm{A}, \mathrm{T}^{30}$ Again, Eq. (10) holds almost per- 


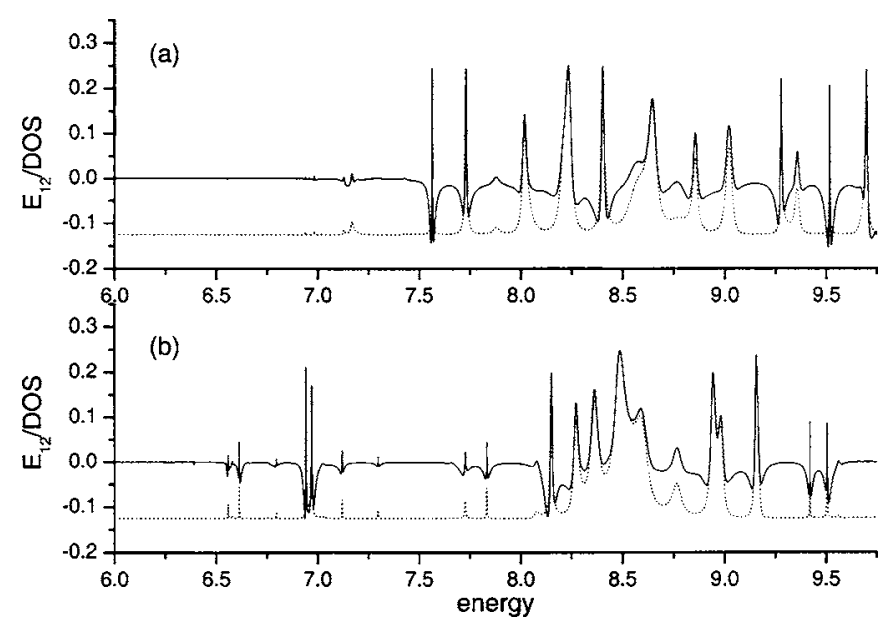

FIG. 3. The weighted emittance (solid line) vs energy for (a) $\lambda_{2}$ sequence and (b) the human chromosome 22 . Dotted line is the quantity $(3 \mathcal{T}-1) / 8$. The units are the same as those of Fig. 1.

fectly for all these different sequences. We emphasize that Eq. (10) is an approximate expression for $\operatorname{poly}(\mathrm{d} \alpha \mathrm{d} \beta)$ sequences but $\bar{E}_{12}=1 / 4$ at $\mathcal{T}=1$ is a universal feature. We have tested Eq. (10) for other types of model DNA sequences: Fibonacci poly-CG sequence, $\lambda_{1}$ chain [the first 60 base pairs (bps) of the $\lambda$ phase sequence], $\lambda_{2}$ chain (the next $60 \mathrm{bps}$ ), $\lambda_{3}, \lambda_{4}$, and $\lambda_{5}$, and the human chromosome 22. Some examples are shown in Fig. 3 where the weighted emittance of $\lambda_{2}$ sequence and that of the human chromosome 22 versus energy at zero temperature are displayed. In general, we found that Eq. (10) is very good for the weighted emittance with large inductivelike dynamic response, but it is not suitable for small positive weighted emittance as well as those with capacitivelike responses.

Now we study the temperature dependence of the emittance. Since the measured quantity is taken at finite time intervals, we have averaged the emittance as well as the global DOS over 100000 realizations of the twist angles. Figure 4(a) shows the weighted emittance for poly $(\mathrm{dGdC})$ at finite temperature $\bar{E}_{12} \equiv\left\langle E_{12}\right\rangle /\langle d N / d E\rangle$ versus energy at different

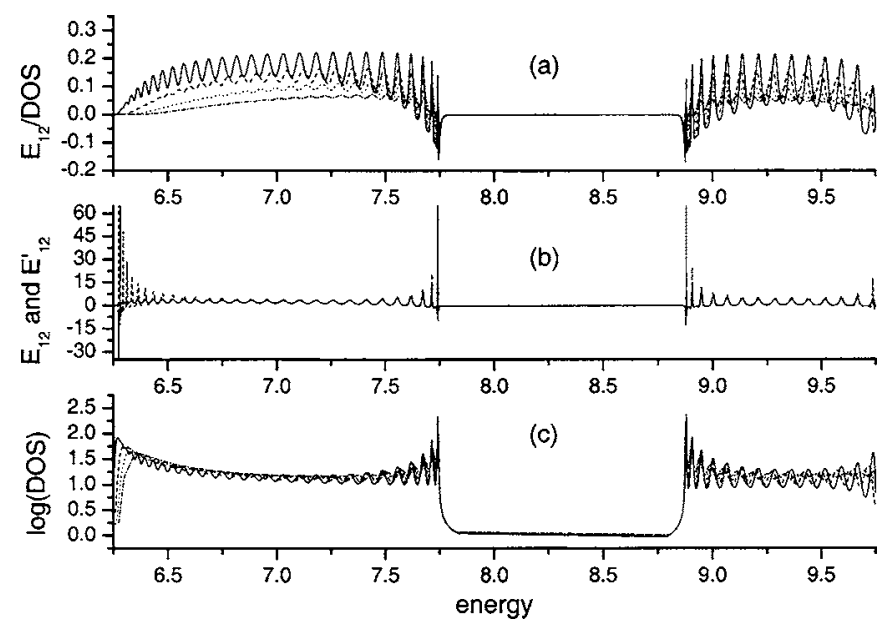

FIG. 4. (a) The weighted emittance and (c) the logarithm of the global DOS vs energy at different temperatures $[T=10 \mathrm{~K}$ (solid line), $T=20 \mathrm{~K}$ (dashed line), $T=30 \mathrm{~K}$ (dotted line), and $T=40 \mathrm{~K}$ (dash-dotted line)]. (b) Emittances $E_{12}$ and $E_{12}^{\prime}$ vs energy at $T=10 \mathrm{~K}$.

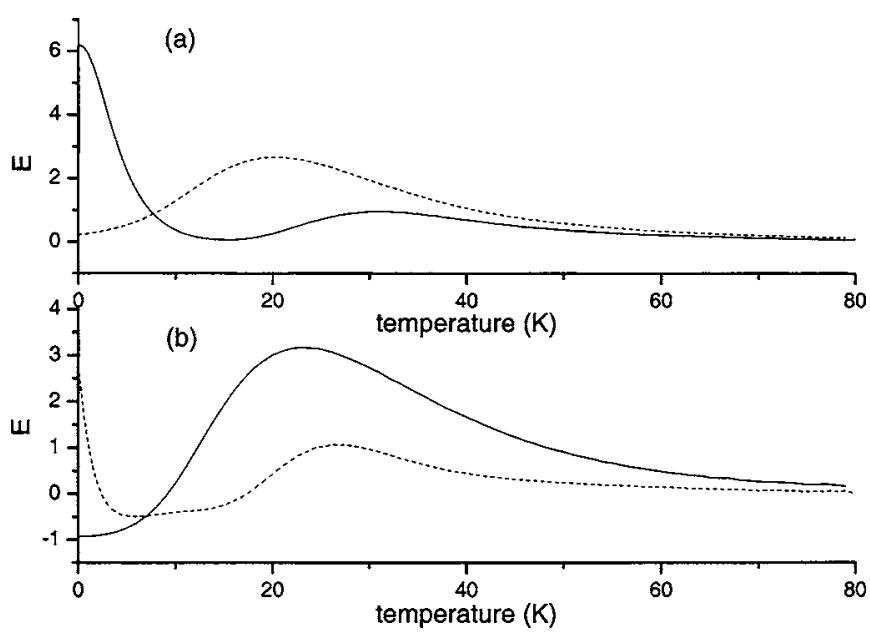

FIG. 5. Emittance vs temperature at different energies: (a) $E=9.612 \mathrm{eV}$ (solid line) and $E=9.12 \mathrm{eV}$ (dotted line); (b) $E=8.94 \mathrm{eV}$ (solid line) and $E=9.694 \mathrm{eV}$ (dotted line).

temperatures. The temperature has two main effects. First, it destroys quantum interference and reduces the weighted emittance. The influence of structural fluctuation due to temperature on emittance is the strongest near the left and right band edges $E=6.2 \mathrm{eV}$ and $9.7 \mathrm{eV}$. As the temperature is increased to $T=10 \mathrm{~K}$, the response of poly $(\mathrm{dGdC})$ near the left band edge becomes inductivelike instead of capacitivelike and the oscillation of emittance vanishes. In the neighborhood of the band edges in the center region $E \sim 7.7 \mathrm{eV}$ and $8.8 \mathrm{eV}$, the influence of temperature is smaller. Second, increasing temperature shifts the peak positions of the emittance. As shown in Fig. 4(a), this shift is toward the band gap in a nonuniform fashion. Near the band gap, the peak positions do not change. The further the peak position is away from the band gap, the larger the shift. The origin of this shift can be understood as follows. The rms value of the hopping matrix element $t_{T}=t_{\text {DNA }} \cos (\sqrt{T / 250 \mathrm{~K}})$ decreases as the temperature rises. For a closed system, different hopping matrix elements give different energy spectra. When this system becomes open, these energy levels evolve into resonant levels. ${ }^{31}$ Hence the peak positions of the emittance change accordingly as the temperature varies. To verify this picture, we have calculated the emittance $E_{12}^{\prime}$ by replacing the fluctuating hopping matrix element $t_{\mathrm{DNA}} \cos (\theta)$ by its rms value $t_{T}$ so that the random fluctuation of $t_{\mathrm{DNA}}$ is removed. Figure 4(b) shows the weighted emittances $\left\langle E_{12}\right\rangle /\langle\mathrm{DOS}\rangle$ and $\left\langle E_{12}^{\prime}\right\rangle /\langle\mathrm{DOS}\rangle$ versus energy. We see that, indeed, the shifting of the peak position is due to the changing of the rms of hopping matrix element while the thermal fluctuation suppresses the quantum interference, resulting to a lower peak height. Our numerical result shows that the sharper the peak, the larger the suppression. For the averaged global DOS, Fig. 4(c) shows that it is less sensitive to the temperature as compared with the weighted emittance. Similar shifting of the peak positions for DOS is observed.

The emittance as a function of temperatures at fixed chemical potentials has interesting behavior, and different behaviors can be observed at different chemical potentials. Figure 5(a) plots the enhancement of the emittance as the 
temperature is increased. At $E=9.612 \mathrm{eV}$ (solid line) which is near the right band edge [see Fig. 1(a)], the emittance is maximum at $T=0$. As the temperature is increased, the emittance drops quickly due to thermal fluctuations. However, a thermal enhancement is possible, indicated by a small peak at $T=30 \mathrm{~K}$ due to shifting of resonant levels by temperature. At $E=9.12 \mathrm{eV}$, the emittance is minimum at $T=0$ (dashed line). As the temperature increases, it rises quickly due to the thermal enhancement, reaches peak value at $T=20 \mathrm{~K}$, and then decays to zero due to the thermal fluctuation. Temperature can also change the response nature of the model DNA sequence. Figure 5(b) shows that at $E=8.94 \mathrm{eV}$, the emittance is negative at $T=0$, exhibiting a capacitivelike behavior. Increasing temperature, the model DNA sequence gives an inductivelike response. This change of dynamic response can be observed for up to $T=30 \mathrm{~K}$. At $E=9.694 \mathrm{eV}$, the system gives an inductivelike response at $T=0$, changes to capacitivelike near $T=10 \mathrm{~K}$, and finally returns to inductivelike behavior at higher temperatures. This result suggests that in the low temperature regime, temperatures can be used as an extra handle to tune the dynamic response of DNA sequences. We emphasize that Eq. (2) is a low frequency expansion. The expansion is appropriate when $\hbar \omega / E_{0} \ll 1$ where $E_{0}$ is some characteristic energy scale of the system. For the DNA sequence we considered, the typical energy scale can be identified using averaged level spacing. Roughly speaking this average spacing is around $0.01 \mathrm{eV}$ (or bigger) which is equivalent to terahertz frequency. It is safe to use Eq. (2) in the gigahertz regime. The influence of contact on dc conductance has been studied in Ref. 15. Basically the influence of contact can be modeled by the coupling between electrode and the scattering region. Different coupling constants can lead to the tunneling regime and transmissive regime, both of them can be found in experimental literatures. ${ }^{15}$ As for the influence of contact on ac transport, we have tried different coupling constants between electrodes and scattering region $t_{d}$. We found that our main conclusion is unchanged. Finally, ab initio calculation (Ref. 24) suggests that $t_{\mathrm{DNA}}=0.4 \mathrm{eV}$. We have tried this value in our study as well. Our conclusion remains in this case.

\section{SUMMARY}

The emittance of model DNA sequences is found to have interesting behavior as a function of electron energy and temperature. An approximated expression of emittance, Eq. (10), is found to give good description of the emittance for several model DNA sequences including poly $(\mathrm{d} \alpha \mathrm{d} \beta)$ where $\alpha, \beta=\mathrm{G}, \mathrm{C}, \mathrm{A}, \mathrm{T}$. For other model sequences such as the Fibonacci poly-CG sequence, $\lambda$ chains, and the human chromosome 22, Eq. (10). fits the emittance very well in the regime of inductivelike response. The results suggest that temperature can have substantial effect on emittance through shifting the resonance levels and smearing of quantum interference. The former may enhance emittance at certain temperature ranges, while the latter reduces emittance value. In the low temperature regime $(T<40 \mathrm{~K})$, the temperature can change the dynamic response of the model DNA sequences from inductivelike to capacitivelike and vice versa.

\section{ACKNOWLEDGMENTS}

The authors gratefully acknowledge support by a RGC grant from the SAR Government of Hong Kong under Grant No. HKU 7044/04P. One of the authors (Z.M.) is supported by NNSFC with Grant Nos. 90103027 and 10274069. Another author (H.G.) is supported by NSERC of Canada, FQRNT of Québec, and CIAR. The computer center of the University of Hong Kong is gratefully acknowledged for the high-performance computing assistance.

${ }^{1}$ D. D. Eley and D. I. Splivey, Trans. Faraday Soc. 58, 441 (1962).

${ }^{2}$ D. B. Hall, R. E. Holmlin, and J. K. Barton, Nature (London) 382, 731 (1996); S. M. Gasper and G. B. Schuster, J. Am. Chem. Soc. 119, 12762 (1997); F. D. Lewis, T. Wu, Y. Zhang, R. L. Letsinger, S. R. Greenfield, and M. R. Wasielewski, Science 277, 673 (1997).

${ }^{3}$ N. C. Seeman, Nature (London) 421, 427 (2003).

${ }^{4}$ E. Braun, Y. Eichen, U. Sivan, and G. Ben-Yoseph, Nature (London) 391, 775 (1998).

${ }^{5}$ H. Fink and C. Schönenberger, Nature (London) 398, 407 (1999).

${ }^{6}$ H. Watanabe, C. Manabe, T. Shigematsu, K. Shimotani, and M. Shimizu, Appl. Phys. Lett. 79, 2462 (2001).

${ }^{7}$ A. Y. Kasumov, M. Kociak, S. Guéron, B. Reulet, V. T. Volkov, D. V. Klinov, and H. Bouchiat, Science 291, 280 (2001).

${ }^{8}$ L. Cai, H. Tabata, and T. Kawai, Appl. Phys. Lett. 77, 3105 (2000).

${ }^{9}$ Y. Zhang, R. H. Austin, J. Kraeft, E. C. Cox, and N. P. Ong, Phys. Rev. Lett. 19, 198102 (2002).

${ }^{10}$ R. Bruinsma, G. Grüner, M. R. D’Orsogna, and J. Rudnick, Phys. Rev. Lett. 85, 4393 (2000).

${ }^{11}$ Z. G. Yu and X. Song, Phys. Rev. Lett. 86, 6018 (2001).

${ }^{12}$ X. Li and Y. Yan, Appl. Phys. Lett. 79, 2190 (2001).

${ }^{13}$ S. Roche, Phys. Rev. Lett. 91, 108101 (2003).

${ }^{14}$ M. Unge and S. Stafstrom, Nano Lett. 3, 1417 (2003).

${ }^{15}$ Y. Zhu, C.-C. Kaun, and H. Guo, Phys. Rev. B 69, 245112 (2004).

${ }^{16}$ W. Ren, J. Wang, Z. Ma, and H. Guo, Phys. Rev. B 72, 035456 (2005).

${ }^{17}$ P. J. de Pablo, F. Moreno-Herrero, J. Colchero, J. G. Herrero, P. Herrero, A. M. Baró, P. Ordejón, J. M. Soler, and E. Artacho, Phys. Rev. Lett. 85, 4992 (2000).

${ }^{18}$ A. J. Storm, J. van Noort, S. de Vries, and C. Dekker, Appl. Phys. Lett. 79, 3881 (2001).

${ }^{19}$ P. Tran, B. Alavi, and G. Gruner, Phys. Rev. Lett. 85, 1564 (2000).

${ }^{20}$ G. Cuniberti, L. Craco, D. Porath, and C. Dekker, Phys. Rev. B 65, 241314 (2002).

${ }^{21}$ For discussions on current conserving and gauge invariance, see M. Büttiker and T. Christen, in Quantum Transport in Semiconductor Submicron Structures, edited by B. Kramer (Kluwer Academic, Dordrecht, 1996), p. 263; M. Büttiker, J. Phys.: Condens. Matter 5, 9361 (1993); A. Prêtre, H. Thomas, and M. Buttiker, Phys. Rev. B 54, 8130 (1996); Z. S. Ma, J. Wang, and H. Guo, ibid. 57, 9108 (1998); 59, 7575 (1999).

${ }^{22}$ V. Gasparian, T. Christen, and M. Buttiker, Phys. Rev. A 54, 4022 (1996).

${ }^{23}$ J. Wang and H. Guo, Phys. Rev. B 54, R11090 (1996); Q. R. Zheng, J. Wang, and H. Guo, ibid. 56, 12462 (1997); C. C. Wan, J. L. Mozos, J. Wang, and H. Guo, ibid. 55, R13393 (1997).

${ }^{24}$ H. Sugiyama and I. Saito, J. Am. Chem. Soc. 118, 7063 (1996); H. Y. Zhang, X. Q. Li, P. Han, X. Y. Yu, and Y. J. Yu, J. Chem. Phys. 117, 4578 (2002).

${ }^{25}$ D. Porath, A. Bezryadin, S. de Vries, and C. Dekker, Nature (London) 403, 635 (2000).

${ }^{26}$ Ch. Adessi and M. P. Anantram, Appl. Phys. Lett. 82, 2353 (2003); Ch. Adessi, S. Walch, and M. P. Anantram, Phys. Rev. B 67, 081405 (2003).

${ }^{27}$ We assumed that the DNA sequence is not gated (zero capacitance limit).

${ }^{28}$ T. Gramespacher and M. Buttiker, Phys. Rev. B 56, 13026 (1997).

${ }^{29}$ D. S. Fisher and P. A. Lee, Phys. Rev. B 23, 6851 (1981).

${ }^{30}$ We find that the transmission coefficient and the weighted emittance for the poly(dAdT) and poly(dAdC) sequence are almost the same.

${ }^{31}$ J. Wang, Y. J. Wang, and H. Guo, Appl. Phys. Lett. 65, 1793 (1994). 